there have been only a few more than a dozen reports including descriptions of $\mathrm{HCV}$ recombinants. However, the frequency of recombination may have been underestimated because not all known HCV recombinants are screened for in routine practice. However, the development of bioinformatics technologies allows for effective screening of genomic sequences for the presence of recombination signals.

Recombination analysis was performed with the Recombinant Detection Program (RDP) version 4.61. This tool provides statistical evidence for the breakpoint site by using six methods (RDP, Geneconv, maximum chisquare, Chimaera, SiScan and 3-seq). A recombination analysis was conducted on 237 complete genome sequences of genotype $2 \mathrm{HCV}$ strains, extracted from the ViPR database. The analysis by the RDP was phylogenetically corroborated by NeighborNet method using the SplitsTree 4.14.1 program and statistically confirmed by the PhiTest method.

Using the RDP method, 116 unique recombination events with a high degree of reliability $(p<0.01)$ were found in the strains studied. The presence of recombination was confirmed by phylogenetic reconstructions. Some of these events occur in dozens of strains, including those belonging to several different subtypes, which may indicate their ancient origin. Futhermore, many strains contain more than one recombination site, and in some cases these areas can overlap. Analysis of parental strains showed that recombinations occur both within and between subtypes, and parental strains often come from different geographic regions. These results may indicate that genotype 2 hepatitis $\mathrm{C}$ virus strains have a higher potential for recombination variability than it was thought before.

\subsection{2} doi: 10.15789/2220-7619-2018-4-7.12

\section{LOW HEPATITIS B VIRUS DNA BURDEN DOES NOT ALWAYS PROTECT FROM LIVER CANCER DEVELOPMENT}

\section{P. Pineau}

Unité “Organisation nucléaire et oncogenèse”, INSERM U993, Institut Pasteur, Paris, France

Due to the considerable number of patients chronically infected with hepatitis B virus (HBV) worldwide (260 million), persistent infection with this agent still represents the principal etiology of the main form of primary liver cancer, hepatocellular carcinoma (HCC). Two decades ago, it has been shown in Far-Eastern countries, that the risk of HCC development increases considerably when high loads $(>2.0 \mathrm{E}+04 \mathrm{IU} / \mathrm{mL})$ of circulating HBV DNA are measured in the plasma of the patients. This observation prompted the release of therapeutic guidelines recommending to treat the patients when HBV DNA exceeds $2.0 \mathrm{E}+03 \mathrm{IU} / \mathrm{mL}$. We recently conducted a molecular analysis on Peruvian patients with HCC infected with HBV genotype F $(n=53)$. Half of these patients were remarkably young $(<40$ years) and their livers unscathed with cirrhosis. Two-third of them were carrying HBV surface antigen, while the remaining were occultly infected. Remarkably, a single patient was displaying HBV DNA loads above the threshold for mandatory treatment. In tumor and non-tumor liver tissues, HBV DNA copy number per cell was usually very low $(0.1-10 \%$ of infected cells) and no clonal integration was detectable. HBV genome mutations usually observed at this stage of the liver disease (Stop precore, basal core promoter double mutations, pre-S deletions) were infrequent $(<25 \%$ of cases $)$ in this series.
In tumor cells, innate immune response was inactive while most DNA repair systems were strongly activated. Our data suggest that for some populations such as American Indians, or in case of infection with specific $\mathrm{HBV}$ strains such as genotype $\mathrm{F}$, the standard procedures of surveillance of patients at risk for HCC are ineffective. Our observations imply that the local patho-physiological context should prevail above guidelines generated from analyses conducted on populations with different geo-anthropological backgrounds. In addition, our observation is somehow reminiscent of the HCC presentation described in Yupik people from Alaska who are also infected with HBV genotype $\mathrm{F}$, the endemic genotype of the Americas. It suggests that similar adverse situations might affect populations with American Indian ancestry. In that respect, research on populations living in Siberia, on the other side of Bering strait, might provide some interesting comparison points. Larger surveys should now be conducted to confirm and refine these preliminary observations.

7.13 doi: 10.15789/2220-7619-2018-4-7.13

\section{THE PREVALENCE OF HIV-1 DRUG RESISTANCE MUTATIONS IN PATIENTS WITH LOW ADHERENCE TO ANTIRETROVIRAL THERAPY IN THE LENINGRAD REGION}

\section{A.N. Shchemelev, Yu.V. Ostankova, D.E. Valutite \\ St. Petersburg Pasteur Institute, St. Petersburg, Russia}

Against the backdrop of an increase in the number of people embarking on antiretroviral therapy (ARVT), the manifestation of drug resistance of HIV is becoming an increasing obstacle to the fight against the epidemic.

Variants of the virus that have drug resistance are usually able to accumulate in the body in the event of interruptions in the intake of antiretroviral drugs. Therefore adherence to therapy is one of the most important factors in the formation of HIV resistance.

The aim of this work is to study the structure of mutations in the HIV genome associated with drug resistance in patients with low adherence.

Blood plasma samples from 269 patients were examined for the detection of mutations in drug resistance. Previously, the history of patients was studied: sex, viral load during therapy, adherence to the latter.

Among patients there are $139(51.67 \%)$ people with low adherence to therapy. In the aggregate of patients with low adherence, men predominate $(57.55 \%)$. This may be due to the peculiarity of the psyche, more typical for male patients. It is also important to note that majority of patients with low adherence belong to disadvantaged groups of people: people who use alcohol and drugs that do not have a permanent place of residence, etc. As a result of an unstable lifestyle, such patients most often interrupt therapy. This, in turn, leads to changes in ARVT regimens, which together with a high viral load (74\% of patients with viral load exceeding 10000 copies/ $\mathrm{ml}$ ) is a factor contributing to the formation of drug resistance.

One of the reasons for the drug resistance of HIV is the appearance of mutations in parts of the virus genome associated with the synthesis of viral enzymes, which are the main targets of therapy. Analysis of the results of studies on the presence of HIV drug resistance revealed several common mutations: M184V (51.08\%), K103N (18.71\%), L74V (12.95\%), K101E (11.51\%), A62V and G190S (10.79\%), the remaining mutations occur in less than $10 \%$ of cases. 